Grundlagen der Kommunikation und Kognition Foundations of Communication and Cognition Bibliotheksausgabe/Library Edition

Herausgeber/Editors

Roland Posner, Georg Meggle 



\section{Meaning Scepticism}

edited by

Klaus Puhl

$W$
$\mathrm{DE}$

Walter de Gruyter - Berlin · New York 1991 
(0) Gedruckt auf säurefreiem Papier, das die US-ANSI-Norm über Haltbarkeit erfüllt.

Printed on acid-free paper which falls within the guidelines of the ANSI to ensure permanence and durability

\section{Library of Congress Cataloguing-in-Publication Data}

Meaning scepticism / edited by Klaus Puhl.

p. $\quad \mathrm{cm}$. - (Foundations of communication and cognition = Grundlagen der Kommunikation und Kognition)

ISBN 0-89925-522-1 : DM 160.00 (est.). ISBN 0-89925-522-1 (U.S.A.)

1. Meaning (Philosophy) 2. Scepticism. 3. Wittgenstein, Ludwig, 1889-1951. 1. Puhl, Klaus, 1954- . II. Series: Foundations of communication and cognition. B840.M457 1991 $121^{\prime} .68-\mathrm{dc} 20$

Meaning scepticism / ed. by Klaus Puhl. - Library ed. - Berlin

; New York : de Gruyter, 1991

(Foundations of communication and cognition)

ISBN 3-11-011833-5

NE: Puhl, Klaus [Hrsg.]

(C) Copyright 1991 by Walter de Gruyter \& Co., D-1000 Berlin 30

Dieses Werk einschließlich aller seiner Teile ist urheberrechtlich geschützt. Jede Verwertung außerhalb der engen Grenzen des Urheberrechtsgesetzes ist ohne Zustimmung des Verlages unzulässig und strafbar. Das gilt insbesondere für Vervielfältigungen, Übersetzungen, Mikroverfilmungen und die Einspeicherung und Verarbeitung in elektronischen Systemen.

Printed in Germany

Satz und Druck: Arthur Collignon GmbH, Berlin 30

Buchbinderische Verarbeitung: Lüderitz \& Bauer, Berlin 61 